\title{
Editorial: Early Events During Host Cell-Pathogen Interaction
}

\author{
Patrícia S. T. Veras ${ }^{1 *}$, Albert Descoteaux ${ }^{2}$, Maria Isabel Colombo ${ }^{3}$ \\ and Juliana P. B. de Menezes ${ }^{1}$ \\ ${ }^{1}$ Laboratory of Parasite-Host Interaction and Epidemiology, Gonçalo Moniz Institute, Salvador, Brazil, ${ }^{2}$ Centre Armand- \\ Frappier Santé Biotechnologie, Institut National de la Recherche Scientifique (INRS), Quebec, QC, Canada, ${ }^{3}$ Instituto de \\ Histología y Embriología de Mendoza (IHEM), Consejo Nacional de Investigaciones Científicas y Técnicas (CONICET)- \\ Universidad Nacional de Cuyo, Mendoza, Argentina
}

Keywords: early immune response, microoorganism, disease control, disease progression, immunotherapeutic interventions

Editorial on the Research Topic

Early Events During Host Cell-Pathogen Interaction

\section{INTRODUCTION}

The outcome of diseases caused by microbial pathogens (Torgerson et al., 2015) depends on the nature of the pathogen and initial host immune response (Aderem and Ulevitch, 2000; Liu and Uzonna, 2012). This Research Topic "Early Events During Host Cell-Pathogen Interaction"

\section{OPEN ACCESS}

Edited and Reviewed by: William Clark Gause,

Rutgers, The State University of New Jersey, United States

*Correspondence:

Patrícia S. T. Veras patricia.veras@fiocruz.br

Specialty section: This article was submitted to Microbes and Innate Immunity, a section of the journal

Frontiers in Cellular and Infection Microbiology

Received: 14 March 2021 Accepted: 05 May 2021 Published: 19 May 2021

Citation:

Veras PST, Descoteaux A, Colombo MI and P. B. de Menezes J (2021) Editorial: Early Events During Host Cell-Pathogen Interaction. Front. Cell. Infect. Microbiol. 11:680557. doi: 10.3389/fcimb.2021.680557 includes 1 review and 1 minireview, and 8 original research articles in the investigation of early events of host immune response against microbial infection.

Toxoplasma gondii, Plasmodium spp., Leishmania spp., and helminths, activate the interleukin33 (IL-33) pathway, known as the 'alarmin' route (Tonacci et al., 2019). Nathan et al. revise the crucial role played by the IL-33 axis in host immune response to these parasites by priming the immune system toward a strong $\mathrm{T}$ helper type-2 (Th2) response that subsequently promotes pathogen clearance. Alternatively, IL-33 can also participate in infection exacerbation. Additionally, these authors provide insight into novel immunotherapeutic interventions by modulating early host immune response using antibodies against IL-33 pathway elements. The essential role of autoantibodies in malaria continues to be debated (Hogh et al., 1994; Guiyedi et al., 2007). Another review published in this Research Topic by Mourão et al. summarizes recent findings regarding autoimmune response in malaria. Mainly, the authors discuss controversies surrounding autoantibody response that contribute to disease pathogenesis or protection.

Among food-borne diseases, amebiasis, caused by the protozoan Entamoeba histolytica, affects approximately 100 million people worldwide (Haque et al., 2003; Lozano et al., 2012). The parasite life cycle involves inter-conversion between trophozoite, that colonizes the mammalian gut, and cyst, which allows disease transmission through the ingestion of contaminated food or water (Haque et al., 2003). The molecular mechanisms governing parasite life cycle remain poorly studied, and cues triggering stage conversion remain unelucidated (Aguilar-Diaz et al., 2010). Singh's group previously identified a novel Entamoeba transcription factor, Encystation Regulatory Motif-Binding Protein (ERM-BP), which contributes to encystation (Manna et al., 2018). Manna et al. further characterized ERM-BP by evaluating its DNA binding functions and nicotinamidase domains in vivo. The overexpression of ERM-BP mutants revealed that this protein is an early regulator of development and heat shock response during Entamoeba infection. Clonorchis sinensis causes 
another zoonotic food-borne parasitic disease that can infect mammals via the ingestion of raw or undercooked fresh fish and shrimp, eventually resulting in host death (Tang et al., 2016). Yan et al. demonstrated that TLR4 might be involved in C. sinensis infection in a resistant mouse strain, C57BL/10. The absence of TLR4 in C. sinensis-infected mice led to severe inflammation in the liver, bile duct proliferation, and biliary and hepatocellular injury. TLR4def mice immune response exhibited M2-like macrophages, a robust Th2 and a reduced Th1 responses.

Intracellular protozoan parasites infect millions of people worldwide. These parasites can cause severe forms of diseases that may result in death. Among vector-borne diseases, Chagas disease, caused by Trypanosoma cruzi, and leishmaniasis by Leishmania spp. are considered the most important due to severity and global distribution (Tang et al., 2016; OMS. Organización Mundial de la Salud, 2019). T. cruzi chronicity and persistence depend on parasitic capacity to escape host control mechanisms (OMS, 2020). Gutierrez et al. compared the effect of parasite infection on immune modulation by bone marrowderived dendritic cells (DCs) and epithelial DCs in vitro. These authors found that DC can facilitate infection progression depending on parasite growth phase and DC maturation. Following T. cruzi invasion, the formation and maturation of parasitophorous vacuoles (TcPV) occurs. Host Rab GTPases are proteins that regulate intracellular vesicular trafficking. Salassa et al. found that endocytic Rabs, including Rab22a, Rab5, and Rab21, are selectively recruited to TcPV at early times of infection, followed by Rab7 and Rab39a at later stages. However, recycling and secretory Rabs are not recruited to the TcPV membrane. The contribution of these proteins to successful T. cruzi infection was demonstrated using cells transfected with mutated endocytic Rab genes.

Although dogs are considered the main reservoir of visceral leishmaniasis caused by L. infantum, most knowledge regarding host-parasite interaction stems from murine models (Cestari et al., 2012; Teixeira-Neto et al., 2014). Nadaes et al. evaluated the potential of DH82, a canine macrophage cell line, response to Leishmania infection. A comparison of the responses of DH82 and RAW 264.7 mouse macrophages against L. infantum promastigotes revealed that both cell lines similarly supported parasite replication/survival. However, notable differences were found in cytokine production, arginase activity and the release of nitric oxide, providing a model to elucidate several aspects of host cell immune response.

Lipophosphoglycan (LPG) is an abundant Leishmania promastigote surface molecule that contribute to parasite phagocytosis and survival within host cells (Mosser and Rosenthal, 1993; Descoteaux and Turco, 1999). When duplication of the LPG2 gene in the L. infantum genome impeded gene targeting by conventional homologous recombination, Jesus-Santos et al. used CRISPR/Cas9

\section{REFERENCES}

Aderem, A., and Ulevitch, R. J. (2000). Toll-Like Receptors in the Induction of the Innate Immune Response. Nature 406, 782-787. doi: 10.1038/35021228 technology to generate $\Delta l p g 2$ mutants that displayed impaired capacity to infect neutrophils, reinforcing the role of LPG as a virulence factor.

The apicomplexan parasite $T$. gondii causes worldwide foodborne infections in mammals following the consumption of contaminated water or raw fruits or vegetables (Guha-Niyogi et al., 2001; Flegr et al., 2014). T. gondii oocysts can cause infections in mice in the absence of digestive factors, differentiating into replicative tachyzoites following phagocytosis by macrophages (Shapiro et al., 2019). Ndao et al. characterized the dynamics of oocyst phagocytosis at the singlecell level. The improved development of tachyzoites in macrophages challenged with free sporocysts or sporozoites compared to whole oocysts suggests that oocyst wall disruption hampers sporozoite excystation in macrophages. Once internalized, T. gondii reprograms host gene expression, including the up-regulation of mTOR-dependent host mRNA translation, resulting in the establishment of infection (Al-Bajalan et al., 2017). In addition to the mTOR-4E-BP1/2 axis, MAPKinteracting kinases 1 and $2(\mathrm{MNK} 1 / 2)$ control the activity of eIF4E, a mRNA cap-binding protein (Leroux et al., 2018). Leroux et al. showed that $T$. gondii inhibits the phosphorylation of axis downstream targets, MNK1/2 and eIF4E, in mouse macrophages. These authors also demonstrated higher $T$. gondii replication in macrophages mutated at the eIF4E phosphorylation residue $v s$. WT cells. Of note, mutant mice were more susceptible to acute toxoplasmosis and showed exacerbated levels of IFN $\gamma$. In all, these data suggest that the MNK1/2-eIF4E axis is required to control $T$. gondii infection, and that its inactivation can be exploited by parasites to promote their survival.

In sum, this Research Topic gives insight into the complexity of host early interactions with various microorganisms, providing information about recent advances on the initial immune response that triggers mechanisms, which result in diseases' control or progression and identifying targets for future interventions on parasitic infections.

\section{AUTHOR CONTRIBUTIONS}

PV wrote the first draft and $\mathrm{AD}, \mathrm{MC}$ and JM edited and commented on the draft. All authors contributed to the article and approved the submitted version.

\section{FUNDING}

PV holds a grant from $\mathrm{CNPq}$ for productivity in research (307832/2015-5).

Aguilar-Diaz, H., Diaz-Gallardo, M., Laclette, J. P., and Carrero, J. C. (2010). In Vitro Induction of Entamoeba Histolytica Cyst-Like Structures From Trophozoites. PloS Negl. Trop. Dis. 4, e607. doi: 10.1371/ journal.pntd.0000607 
Al-Bajalan, M. M. M., Xia, D., Armstrong, S., Randle, N., and Wastling, J. M. (2017). Toxoplasma Gondii and Neospora Caninum Induce Different Host Cell Responses At Proteome-Wide Phosphorylation Events; a Step Forward for Uncovering the Biological Differences Between These Closely Related Parasites. Parasitol. Res. 116, 2707-2719. doi: 10.1007/s00436-017-5579-7

Cestari, I., Ansa-Addo, E., Deolindo, P., Inal, J. M., and Ramirez, M. I. (2012). Trypanosoma Cruzi Immune Evasion Mediated by Host Cell-Derived Microvesicles. J. Immunol. 188, 1942-1952. doi: 10.4049/jimmunol.1102053

Descoteaux, A., and Turco, S. J. (1999). Glycoconjugates in Leishmania Infectivity. Biochim. Biophys. Acta 1455, 341-352. doi: 10.1016/s0925-4439(99)00065-4

Flegr, J., Prandota, J., Sovickova, M., and Israili, Z. H. (2014). Toxoplasmosis-a Global Threat. Correlation of Latent Toxoplasmosis With Specific Disease Burden in a Set of 88 Countries. PloS One 9, e90203. doi: 10.1371/ journal.pone.0090203

Guha-Niyogi, A., Sullivan, D. R., and Turco, S. J. (2001). Glycoconjugate Structures of Parasitic Protozoa. Glycobiology 11, 45R-59R. doi: 10.1093/ glycob/11.4.45r

Guiyedi, V., Chanseaud, Y., Fesel, C., Snounou, G., Rousselle, J-C., Lim, P., et al. (2007). Self-Reactivities to the non-Erythroid Alpha Spectrin Correlate With Cerebral Malaria in Gabonese Children. PloS One 2, e389. doi: 10.1371/ journal.pone.0000389

Haque, R., Huston, C. D., Hughes, M., Houpt, E., and Petri, W.A. Jr. (2003). Amebiasis. N Engl. J. Med. 348, 1565-1573. doi: 10.1056/NEJMra022710

Hogh, B., Petersen, E., Crandall, I., Gottschau, A., and Sherman, I. W. (1994). Immune Responses to Band 3 Neoantigens on Plasmodium FalciparumInfected Erythrocytes in Subjects Living in an Area of Intense Malaria Transmission are Associated With Low Parasite Density and High Hematocrit Value. Infect. Immun. 62, 4362-4366. doi: 10.1128/ IAI.62.10.4362-4366.1994

Leroux, L. P., Lorent, J., Graber, T. E., Chaparro, V., Masvidal, L., Aguirre, M., et al. (2018). The Protozoan Parasite Toxoplasma Gondii Selectively Reprograms the Host Cell Translatome. Infect. Immun. 86. doi: 10.1128/IAI.00244-18

Liu, D., and Uzonna, J. E. (2012). The Early Interaction of Leishmania With Macrophages and Dendritic Cells and its Influence on the Host Immune Response. Front. Cell Infect. Microbiol. 2, 83. doi: 10.3389/fcimb.2012.00083

Lozano, R., et al. (2012). Global and Regional Mortality From 235 Causes of Death for 20 Age Groups in 1990 and 2010: A Systematic Analysis for the Global Burden of Disease Study 2010. Lancet 380, 2095-2128. doi: 10.1016/S01406736(12)61728-0
Manna, D., Lentz, C. S., Ehrenkaufer, G. M., Suresh, S., Bhat, A., and Singh, U. (2018). An NAD(+)-dependent Novel Transcription Factor Controls Stage Conversion in Entamoeba. Elife 7. doi: 10.7554/eLife.37912

Mosser, D. M., and Rosenthal, L. A. (1993). Leishmania-Macrophage Interactions: Multiple Receptors, Multiple Ligands and Diverse Cellular Responses. Semin. Cell Biol. 4, 315-322. doi: 10.1006/scel.1993.1038

OMS (2020). Leishmaniasis.

OMS. Organización Mundial de la Salud (2019). La Enfermedad De Chagas (Tripanosomiasis Americana).

Shapiro, K., Bahia-Oliveira, L., Dixon, B., Dumétre, A., de Wit, L. A., VanWormer, E., et al. (2019). Environmental Transmission of Toxoplasma Gondii: Oocysts in Water, Soil and Food. Food Waterborne Parasitol. 15, e00049. doi: 10.1016/ j.fawpar.2019.e00049

Tang, Z. L., Huang, Y., and Yu, X. B. (2016). Current Status and Perspectives of Clonorchis Sinensis and Clonorchiasis: Epidemiology, Pathogenesis, Omics, Prevention and Control. Infect. Dis. Poverty 5, 71. doi: 10.1186/s40249-016-0166-1

Teixeira-Neto, R. G., da Silva, E. S., Nascimento, R. A., Belo, V. S., de Oliveira, C. L., Pinheiro, L. C., et al. (2014). Canine Visceral Leishmaniasis in an Urban Setting of Southeastern Brazil: An Ecological Study Involving Spatial Analysis. Parasit. Vectors 7, 485. doi: 10.1186/s13071-014-0485-7

Tonacci, A., Quattrocchi, P., and Gangemi, S. (2019). Il33/St2 Axis in Diabetic Kidney Disease: A Literature Review. Med (Kaunas) 55. doi: 10.3390/ medicina55020050

Torgerson, P. R., Devleesschauwer, B., Praet, N., Speybroeck, N., Willingham, A. L., Kasuga, F., et al. (2015). World Health Organization Estimates of the Global and Regional Disease Burden of 11 Foodborne Parasitic Diseases, 2010: A Data Synthesis. PloS Med. 12, e1001920. doi: 10.1371/journal.pmed.1001920

Conflict of Interest: The authors declare that the research was conducted in the absence of any commercial or financial relationships that could be construed as a potential conflict of interest.

Copyright (C) 2021 Veras, Descoteaux, Colombo and P. B. de Menezes. This is an open-access article distributed under the terms of the Creative Commons Attribution License (CC BY). The use, distribution or reproduction in other forums is permitted, provided the original author(s) and the copyright owner(s) are credited and that the original publication in this journal is cited, in accordance with accepted academic practice. No use, distribution or reproduction is permitted which does not comply with these terms. 